Genetic association studies

\section{Publishing genetic association studies in Thorax}

\section{J A Wedzicha, I P Hall}

G enetic association studies have the potential to provide a valuable insight into disease mechanisms. However, many studies submitted to journals such as Thorax are underpowered or poorly designed. This has led to a number of journals providing guidance to investigators regarding the design of studies which would be considered for publication within the journal. In the editorial which follows we provide some guidance on the key issues which should be considered in assessing the validity of genetic association studies in respiratory disease. While Thorax will always consider manuscripts which contain informative original research, in general it is unlikely that papers which do not conform to the guidance given in the accompanying editorial will be acceptable for publication unless extenuating circumstances exist.

Thorax 2005:60:357.

doi: 10.1136/thx.2005.043802

\section{Authors' affiliations \\ J A Wedzicha, Editor in Chief, Thorax \\ I P Hall, Associate Editor, Thorax}

Correspondence to: Professor J A Wedzicha Thorax Editorial Office, BMJ Journals, BMA House, Tavistock Square, London WC1H 9JR, UK; j.a.wedzicha@qmul.ac.uk

\title{
Genetic association studies in Thorax
}

\section{P Hall, J D Blakey}

\section{A guide to assessing the validity of genetic association studies in respiratory disease}

ex

ncreasing knowledge regarding the extent of genetic variation in the human genome has led to an explosion of interest in performing genetic association studies in complex diseases. Well designed studies have the potential to provide functionally relevant data on the pathophysiology of disease initiation and severity. ${ }^{1}$ Unfortunately, this field has acquired a bad reputation over recent years because of problems with poor design and variable replication of findings. ${ }^{23}$ Because such studies are relatively easy to undertake when one has access to a population of patients with disease, the number of such studies has increased markedly: Thorax now receives, on average, eight each month. As there are a number of common flaws present in many of these studies, we felt it would be helpful to publish some broad guidance on the subject. While Thorax will always be keen to receive high quality manuscripts dealing with genetic studies in respiratory disease, in the future it is unlikely that submitted manuscripts will be sent out for further review if they do not conform to the guidance contained within this editorial.

\section{STUDY POPULATION SIZE}

The majority of submitted genetic association studies use a case-control design, so this is the focus of this editorial. The limiting factor in recruitment is usually the number of cases available to study. There are some advantages in increasing the number of controls (that is, having more than one matched control for each case): in practice 2:1 matching of controls to cases often provides the most efficient design for relatively common diseases. For any given genetic association study an initial power calculation should be undertaken to determine the power of the study to detect effects. For most of the genetic factors contributing to common complex diseases, published relative risks have been no higher than 2. The size of population required to determine a relative risk of this magnitude will depend upon the allele frequency of the polymorphisms under consideration (table 1). Programs for estimating required sample size are readily available-for example, downloadable from http://hydra.usc.edu/gxe ${ }^{4}$ and online at http://Statgen.iop.kcl.ac.uk/ gpc. $^{5}$

For the majority of genes of interest population sizes of several hundred will be required to ensure adequate power. For many common respiratory diseases such as asthma and chronic obstructive pulmonary disease (COPD), populations of an adequate size are already available in many centres. The investigation of gene-environment or gene-gene interactions greatly increases the sample size requirement (and may necessitate collaboration between several research groups), but is to be encouraged as this has the potential for greater insight into disease. ${ }^{6}$ For common diseases, therefore, it is unlikely that studies involving small numbers of subjects (for example, 150 asthmatics and 150 controls) will be adequately powered to truly estimate population contributions of genetic variants. In general, studies which the journal would wish to publish will either have large sample sizes or alternatively demonstrate replication in two independent populations. This approach may not be practical for very rare conditions and, where a strong case can be made, smaller studies which provide preliminary information on insight into novel mechanisms of disease would still be of interest.

\section{SNP, HAPLOTYPES OR FUNCTIONALLY RELEVANT POLYMORPHISMS?}

The public domain databases contain more than six million single nucleotide polymorphisms (SNPs) and it is likely that there are over 10 million SNPs with allele frequencies greater than $1 \%$. The number of polymorphisms involved in disease aetiology and modulation is therefore massively outweighed by those not involved, which means that association studies using randomly selected SNPs have very high false positive rates. This is compounded by publication bias: negative association studies are more difficult to publish and may not even be written up by investigators. To raise the prior probability of true association, very careful consideration should be given in the initial design with regard to the 
Table 1 Suggested population sizes (of cases) required in a case control study seeking the effect size shown for a given minor allele frequency $(\alpha=0.05$, power $90 \%)$

\begin{tabular}{lllc}
\hline \multirow{2}{*}{$\begin{array}{l}\text { Minor allele } \\
\text { frequency }\end{array}$} & GRR sought & Cases required & \\
\cline { 3 - 4 } & 1.5 & Dominant effect & Recessive effect \\
\hline 0.01 & 2 & 5500 & $>1000000$ \\
0.05 & 1.5 & 1900 & $>300000$ \\
& 2 & 1300 & 50000 \\
0.2 & 1.5 & 400 & 15000 \\
& 2 & 600 & 5000 \\
0.4 & 1.5 & 220 & 1500 \\
& 2 & 750 & 900 \\
\hline
\end{tabular}

GRR, genotype relative risk

The numbers given assume that the same numbers of controls as cases are to be recruited. Exact numbers will depend on the precise hypothesis under test and the method of analysis: numbers required may be substantially greater (for example, where attempts to control for confounders are made). In these instances input from a statistician is advised.

polymorphisms chosen. Where functional information regarding a polymorphism in a given candidate gene is available, this may help to prioritise selection. If no functional information is available regarding the gene of interest, then the choice is either to undertake functional studies on the polymorphic variants within that gene or to select tag SNPs from which information can be inferred for other variants in that gene. ${ }^{7}$ The alternative is to use combinations of SNPs (or other polymorphisms) across that genetic region (haplotypes): the disadvantage with this approach is that there are likely to be many different haplotypes and hence the population study size will need to be increased accordingly. Many studies submitted to Thorax examine only a single SNP in the gene of interest: unless there are good supporting functional data on the chosen polymorphism, ideally in the same study population, this approach is unlikely to be very informative. In general, investigators interested in pursuing genetic studies for their favoured candidate gene should look carefully at the polymorphic variation at that genetic locus, study the haplotype structure and linkage disequilibrium profile around the region (increasing information will be available in the public domain as a result of the HapMap Project), and evaluate functional effects of known polymorphisms at this locus.

\section{MULTIPLE TESTING}

The issues raised in the preceding section will help prioritise genetic variants worthy of evaluation but it is likely that there will be more than one genetic factor involved in the primary analysis. Similarly, there may be more than one phenotype of interest-for example, a study might consider the presence of asthma as the major phenotype, but might also look at bronchial hyperresponsiveness, IgE, asthma severity, or response to medication as additional phenotypes. Each additional genetic factor and each additional phenotype to be studied adds to the number of comparisons made in the analysis and gives rise to additional problems of multiple testing. Although new methods are increasingly applied, ${ }^{8}$ there is no simple answer to this issue. We hope the following advice may help.

Before commencing the analysis it is critical to determine the primary end point for the study. Sub-analyses can also be reported but it should be made explicitly clear that positive associations have come from secondary analyses when presenting data. Some studies require a more complicated analytical approach-for example, studies on genetic factors influencing the development of COPD in smokers need to allow for known confounding effects on lung function such as age, sex, height, and duration of smoking exposure. This is usually done using regression analysis although alternative approaches (such as those using recursive partitioning) may also be of value in this setting, especially for the investigation of epistasis. ${ }^{9}$

One particular concern for genetic association studies is the repeated use of the same population for different association studies. It may not be apparent to readers of a manuscript that the population has been used for previous analyses. This issue should at least be acknowledged by authors submitting papers describing sequential studies in a given population. Ideally, where multiple candidate genes are to be assessed in the population, it is preferable to report data on all the genes of interest in a single comprehensive manuscript rather than in multiple smaller papers.
The use of an independent replication sample greatly increases the confidence that an observed association is true. One potential approach is to use one sample for hypothesis generation (that is, accepting "significant" $p$ values without correction) and then seeking replication for only those initially associated variables in a second population.

\section{POPULATION STRATIFICATION}

The above sections have dealt with major issues concerning study size and the selection of genetic variants for study. A common flaw is the inappropriate selection of study populations. Failure to match the control and study populations for ethnic or geographical origin may lead to spurious results because of population stratification. Increasingly it has been recognised that even apparently homogenous populations may show sub-stratification.

One should aim to match controls and cases for every characteristic bar the outcome under study. However, bias from the recruitment locations of cases and controls is commonly seen: controls are often attending hospital for another reason, or may be blood donors or younger healthy volunteers. These types of control group may not, by their nature, be representative of the population at large.

With this in mind, it is reassuring to see study populations typed for unlinked markers to identify and address stratification. An alternative approach which is feasible for some conditions is to use family based association approaches. Investigators are directed elsewhere for a fuller discussion of population stratification (for example, Cardon and Palmer $^{10}$ ), but should at least reassure themselves that the control and study populations are drawn from the same general pool.

\section{SUMMARY}

The above issues are some of the most important factors to be taken into consideration when assessing the validity of genetic association studies. Despite the recent criticism of this kind of study, good examples have the potential to provide novel insight into mechanisms of disease. Authors intending to submit a genetic association study to Thorax should consider whether or not their study has addressed the following specific questions:

- Is the study size adequate to provide a reasonable estimate of the population contribution of the genetic variation under consideration?

- Is the control population appropriately selected? 
- Is the choice of polymorphism(s) studied at a given genetic locus logical?

- Has linkage disequilibrium at the relevant genetic locus been considered?

- Are phenotypes well documented?

- Have issues of multiple testing been addressed?

- Have findings been replicated in a second sample or are there functional data to support findings in the main study population?

- Does the genetic association study advance our understanding of the mechanisms underlying the disease of interest or its treatment?

Thorax 2005;60:357-359.

doi: $10.1136 /$ thx. 2005.040790

\section{Authors' affiliations}

J D Blakey, I P Hall, Division of Therapeutics and Molecular Medicine, Queen's Medical

Centre, University Hospital, Nottingham, UK

Correspondence to: Professor I P Hall, Division of Therapeutics and Molecular Medicine, D Floor, South Block, Queen's Medical Centre, University Hospital, Nottingham NG7 2UH,

UK; ian.hall@nottingham.ac.uk

The authors declare no conflict of interest with the material presented in this editorial.

\section{REFERENCES}

1 Risch N, Merikangas K. The future of genetic studies of complex human diseases. Science 1996;273:1516-7.

2 Hirschhorn JN, Lohmueller K, Byrne E, et al. A comprehensive review of genetic association studies. Genet Med 2002;4:45-61.

3 Lohmueller KE, Pearce CL, Pike M, et al. Metaanalysis of genetic association studies supports a contribution of common variants to susceptibility to common disease. Nat Genet 2003;33:177-82.

4 Gauderman WJ. Sample size requirements for matched case-control studies of gene-environment interaction. Stat Med 2002;21:35-50.

5 Purcell S, Cherny SS, Sham PC. Genetic power calculator: design of linkage and association genetic mapping studies of complex traits. Bioinformatics 2003;19:149-50.

6 McClearn GE. Nature and nurture: interaction and coaction. Am J Med Genet 2004; 124B: 124-30

7 Neale BM, Sham PC. The future of association studies: gene-based analysis and replication. Am J Hum Genet 2004;75:353-62.

8 Sabatti C, Service S, Freimer N. False discovery rate in linkage and association genome screens for complex disorders. Genetics 2003;164:829-33.

9 Ritchie MD, Hahn LW, Roodi N, et al. Multifactordimensionality reduction reveals high-order interactions among estrogen-metabolism genes in sporadic breast cancer. Am J Hum Genet 2001;69:138-47.

10 Cardon LR, Palmer U. Population stratification and spurious allelic association. Lancet 2003;361:598-604.

\section{Peripheral muscle training in COPD: still much to learn}

\section{D L Morgan}

\section{We know that physical training can improve general functional exercise performance in COPD but we still do not understand the subtleties of different training modes}

A ctivity limitation and breathlessness are the main clinical features of advanced chronic obstructive pulmonary disease (COPD). During the last few years it has become recognised that this activity limitation relates in some way to peripheral muscle dysfunction that can be partly reversed by the process of pulmonary rehabilitation. ${ }^{12}$ There is still debate about the detailed nature of the peripheral muscle dysfunction but most investigators would agree that deconditioning through inactivity plays a major role. In health, the age related loss of muscle mass can be prevented by maintained activity or reversed by training. In COPD the contribution of other factors to a specific myopathy such as systemic inflammation, hypoxia, or steroid damage remains uncertain. Physical training is the obvious way of improving the function of deconditioned peripheral muscles, although other options such as electrical stimulation or pharmacological treatment can also have an effect. There is now clear evidence that pulmonary rehabilitation programmes that include individually prescribed but generic physical exercise training are capable of improving functional exercise capacity and health status. ${ }^{3}$ Rehabilitation programmes generally contain a number of therapeutic elements, but it is also clear that the overall benefits of the process do not occur in the absence of some form of physical training. In addition, it has also been a consistent finding that the resulting improvements in general exercise performance and health status appear to relate poorly to each other. In most cases the magnitude of the improvement in quality of life is greater than the more modest improvements in general exercise capacity.

There are several possible explanations for this apparent dissociation. Firstly, exercise training may be the vector for improvement in health status through intermediary mechanisms that involve less tangible factors such as confidence, self-efficacy, or motivation. Another explanation lies in our simplistic assumption that just walking a little bit further improves the quality of life. The frustrations of life for people with COPD are possibly more closely related to the interference by dyspnoea in the basic activities of daily living. ${ }^{4}$ Most rehabilitation programmes concentrate on continuous endurance exercise training that can improve walking or cycling ability but may not be expected to have a direct effect on domestic task performance that involves the upper body. Training for upper and lower limb strength may be expected to have a more direct effect on domestic task performance, although as yet there is no evidence to support this presumption. Even if the optimal mode of physical training is uncertain, there is still further inconsistency in the prescription of the dose of exercise required to have the intended effect. In this respect, two recent systematic reviews have helped to summarise our knowledge in this area. The first examines the overall merits of peripheral muscle strength training and the second, in this issue of Thorax, examines the comparative benefits of different training modalities and effect of intensity. ${ }^{56}$

\section{DOES PERIPHERAL MUSCLE STRENGTH TRAINING WORK IN COPD?}

Most training programmes have previously employed endurance training that improves exercise performance but will not have any effect on muscle mass or strength. To improve the latter, specific resistance training may be required. The systematic review by $\mathrm{O}^{\prime}$ Shea $e t a l^{5}$ found nine methodologically acceptable clinical trials that examined the effect of upper or lower limb resistance training on a total of 236 patients. The strength training was performed with free weights or exercise equipment and individually prescribed as repetitions based on a variable fraction (50-85\%) of the one repetition maximum (IRM). Some studies incremented the 
training load through the programme. The generally short term outcomes were recorded in terms of impairment (muscle strength, laboratory exercise capacity), activity (walking tests), or participation (quality of life questionnaires).

The pooled results of the training studies show that fairly rigorous strength training can be achieved in people with advanced COPD. They also demonstrated significant improvements in upper and lower body strength in the trained muscle groups. In spite of the findings of one study that demonstrated equivalent benefits of strength and endurance training on exercise capacity, there remains insubstantial evidence of the ability of strength training alone to regularly improve exercise capacity or health status. ${ }^{7}$ The longer term benefits of isolated strength training or sustainability of domestic maintenance training are also unclear. To date there have been no studies of the effect of pure resistance training on activities of daily living or domestic function.

\section{WHAT TRAINING MODALITIES AND PRESCRIPTION INTENSITIES ARE BENEFICIAL?}

If the wider advantages of strength training are not clear, then it would be useful to learn how it should be applied or whether it should be combined with endurance training to maximise the benefit. This is examined in the current issue of Thorax in a systematic review of the comparison of exercise training modalities and training intensities in patients with COPD by Puhan et al. ${ }^{6}$ This study identified 15 randomised controlled trials where a head to head comparison of exercise modality or training intensity had been made. In the studies which directly compared endurance with strength training, the results were surprisingly inconsistent. In some cases endurance training produced a greater improvement in physical performance than strength training and in others the reverse occurred. However, there does appear to be a stronger influence of strength training on quality of life as reflected in the Chronic Respiratory Questionnaire (CRQ). These finding are different from the systematic review by O'Shea et al and presumably reflect the selection of head to head study group comparisons rather than a usual care control group.

If both strength and endurance training have merits, then it would be sensible to combine these two modalities to enhance the benefit. In five trials where endurance training was compared with the combination of endurance and strength training, the results were slightly disappointing because the additional benefits of added strength in the com- bined training were limited to gains in strength only. There did not appear to be any carry over in terms of improved exercise capacity or health status..$^{8-10}$ Most endurance training regimes are continuous in format and may not be sustainable by patients with severe impairment. Training efficiency can be improved by breaking the exercise sessions up into intervals. ${ }^{11}{ }^{12}$ In such patients the review suggests that this approach may enhance the benefit of training. Comparative trials of training intensity have been uncommon but suggest that greater physiological benefits can be achieved with high intensity ( $80 \%$ peak oxygen consumption) than with low intensity exercise training. ${ }^{13}$ However, some improvements in health status are still seen with lower intensity training. ${ }^{14}$

\section{HOW GOOD IS THE EVIDENCE?}

These systematic reviews have helped us to order our knowledge of peripheral muscle training in COPD. However, the pitfalls of systematic reviews and metaanalyses are exposed by comparison. The incomplete agreement is a reflection of the unmatched selection policies of the reviews. Nevertheless, both reviews agree that patients can adhere to physical training programmes and gain benefit from them. Beyond that, the reviews have exposed significant deficiencies in existing evidence and major gaps in our understanding of the field. Most of the included studies contain small numbers of subjects and have methodological flaws, particularly with regard to blinding of interventions and outcome assessment. It is easy to be critical of these shortcomings but any investigator who has undertaken a training study will appreciate the very difficult challenges that these clinical trials present. Patients who are recruited for rehabilitation trials are often vulnerable and expected to make an active commitment to the training programme over a prolonged period without dropping out. It is virtually impossible to blind the training intervention but outcome assessments can and should be made by blinded assessors.

In the athletic domain, physical training is recognised as a highly complex intervention. Although we have seen the benefits of simple training regimes in pulmonary rehabilitation, we have really only begun to explore the complexity of training science in this area. The benefits of physical training accrue with the combined product of training intensity, session frequency, and duration. Most training also results in a training-specific response rather than more general benefit. The interplay of these factors in determining the total training load is largely unknown. In addition, most of the trials have only examined the short term effects of training and have not observed the longer term results or examined the effects or feasibility of maintenance therapy.

We know that physical training can improve general functional exercise performance in COPD but the improvements are relatively modest and nonspecific. This offers proof of concept but does not allow us to examine the subtleties of different training modes. These need to be explored in association with the appropriate outcome assessments. Combinations of outcomes that include activities of daily living and reflections of domestic function and participation are more germane to the aims of rehabilitation.

Thorax 2005;60:359-360. doi: 10.1136/thx.2004.035998

Correspondence to: Dr M D L Morgan, Institute for Lung Health, Department of Respiratory Medicine and Thoracic Surgery, University Hospitals of Leicester, Glenfield Hospital, Leicester, LE3 9QP, ÚK; mike.morgan@uhl-tr. nhs.uk

\section{REFERENCES}

1 American Thoracic Society. Pulmonary rehabilitation-1999. Am J Respir Crit Care Med 1999: 159:1666-82.

2 British Thoracic Society. Statement on pulmonary rehabilitation. Thorax 2001;56:827-34.

3 Lacasse $Y$, Brosseau L, Milne S, et al. Pulmonary rehabilitation for chronic obstructive pulmonary disease. Cochrane Database Syst Rev 2002;3:CD003793.

4 Elkington $\mathrm{H}$, White $\mathrm{P}$, Addington-Hall J, et al. The last year of life of COPD: a qualitative study of symptoms and services. Respir Med 2004;98:439-45.

5 O'Shea SD, Taylor NF, Paratz J. Peripheral muscle strength training in COPD: a systematic review. Chest 2004; 126:903-14.

6 Puhan MA, Schünemann HJ, Frey M, et al. How should COPD patients exercise during respiratory rehabilitation? Comparison of exercise modalities and intensities to treat skeletal muscle dysfunction. Thorax 2005:60:367-75.

7 Spruit MA, Gosselink R, Troosters T, et al. Resistance versus endurance training in patients with COPD and peripheral muscle weakness. Eur Respir J 2002;19:1072-8

8 Mador MJ, Bozkanat E, Aggarwal A, et al. Endurance and strength training in patients with COPD. Chest 2004; 125:2036-45.

9 Bernard S, Whittom F, Leblanc P, et al. Aerobic and strength training in patients with chronic obstructive pulmonary disease. Am J Respir Crit Care Med 1999;159:896-901.

10 Ortega F, Toral J, Cejudo P, et al. Comparison of effects of strength and endurance training in $\mathrm{pa}-$ tients with chronic obstructive pulmonary disease. Am J Respir Crit Care Med 2002; 166:669-74.

11 Coppoolse R, Schols AM, Baarends EM, et al. Interval versus continuous training in patients with severe COPD: a randomized clinical trial. Eur Respir J 1999;14:258-63.

12 Vogiatzis I, Nanas S, Roussos C. Interval training as an alternative modality to continuous exercise in patients with COPD. Eur Respir J 2002;20:12-9.

13 Casaburi R, Patessio A, loli $F$, et al. Reductions in exercise lactic acidosis and ventilation as a result of exercise training in patients with obstructive lung disease. Am Rev Respir Dis 1991;143:9-18.

14 Normandin EA, McCusker C, Connors M, et al. An evaluation of two approaches to exercise conditioning in pulmonary rehabilitation. Chest 2002;121:1085-91. 
Recovery pathway of post-SARS patients

\section{Recovery pathway of post-SARS patients}

\section{J C K Chan}

\section{The recovery pathway of SARS survivors mirrors that of ARDS patients in several respects}

$\mathrm{T}$ he article by Hui et al in this issue of Thorax ${ }^{1}$ and an earlier article in Thorax by $\mathrm{Ng}$ et $\mathrm{al}^{2}$ on the longer term functional state of patients who have recovered from severe acute respiratory syndrome (SARS) have arrived in a timely fashion for two reasons. Firstly, as survivors of the global epidemic which shocked the world in 2003, patients who have recovered from SARS are certainly an important source of world knowledge on the longer term impact of the illness on the health of those affected. Secondly, there has recently been renewed interest in the long term outcome of patients who have survived acute respiratory distress syndrome (ARDS), as evidenced by seven studies published since 2000 on patient outcome following an episode of ARDS. ${ }^{3-9}$

\section{WHY IS SARS PUT IN THE SAME LIGHT AS ARDS?}

The question of whether SARS is just ARDS has been asked since the 2003 outbreak. ${ }^{10}$ Indeed, the more severe form of SARS does resemble ARDS, with both being a process of acute lung injury extensively involving the lungs, causing severe gas exchange impairment and requiring intensive care and ventilatory support. The recovery potential of the lungs in SARS patients may closely follow the recovery potential of ARDS patients. However, one needs to keep in mind that only about $30 \%$ of SARS patients in the cohorts reported by Hui et al and $\mathrm{Ng}$ et al had spent time in the intensive care unit (ICU), ventilated or otherwise. Any fair comparison of the lung recovery pattern between the two diseases should theoretically be restricted to a comparison between ventilated ARDS patients and ventilated SARS patients, both preferably having been subjected to similar ventilatory strategies to minimise the confounding effect of the well described phenomenon of ventilator-associated lung injury (VALI).

RECOVERY OF LUNG FUNCTION It is therefore reassuring to see in the study by Hui et al that, at 6 months, there was no difference in the lung function parameters between the ICU non-intubated SARS patients and the ICU intubated SARS patients, suggesting either that VALI did not contribute to pulmonary outcome at 6 months or that the present day practice of lung protective strategy was effective in preventing VALI. However, one should be cautioned against reading too much into these data as the numbers of subjects in each arm were small (six and 25, respectively). Indeed, there was a subtle (albeit insignificant) difference in the carbon monoxide transfer factor (TLCO) between the non-intubated ICU group and the intubated group, with a lower median TLCo in the latter. The latter group also had a significantly higher peak level of lactate dehydrogenase which has been reported to be a marker of SARS severity, ${ }^{11}$ rendering a more severe disease as the more likely explanation for the relatively low TLCO at 6 months. VALI did not appear to have any detectable impact on lung recovery potential in the cohort studied by Hui et al.

Based on the lung function findings reported by Hui et al, what is currently known about pulmonary morbidity following ARDS can be applicable to postSARS patients: "pulmonary function returns to normal or is nearly normal by 6 months to 1 year, with the exception of a persistent reduction in carbon monoxide diffusion capacity". ${ }^{3}$ Hui et al reported that $15.5 \%$ of their patients had impaired TLCO and a $7.3 \%$ reduction in lung volumes at 6 months. In the same study about a quarter of the patients were found to have respiratory muscle weakness at 3 months, suggesting extrapulmonary pathology for the reduction in lung volumes. $\mathrm{Ng}$ et al, however, reported a higher proportion of post-SARS patients with pulmonary function impairment at 6 months, with $35 \%$ having impaired TLCO and $28.1 \%$ having a reduction in lung volumes. It is not clear why the patients studied by $\mathrm{Ng}$ et al had more pulmonary sequelae than those studied by Hui et al. A detailed comparison of the two cohorts in terms of severity of illness during the SARS episode, the proportion of ventilated patients, the issue (or non-issue) of VALI, the different reference ranges used for the pulmonary function parameters $^{*}$, as well as the proportion of patients with respiratory muscle weakness may render a crude comparison of the provided figures not advisable. All in all, it does appear that most postSARS patients had a favourable pulmonary outcome similar to post-ARDS patients.

\section{RECOVERY OF FUNCTIONAL CAPACITY AND QUALITY OF LIFE}

Aside from pulmonary considerations, SARS resembles ARDS in yet another important dimension. In a study by Herridge et $a l^{3}$ of post-ARDS patients, the median 6 minute walk distance improved steadily during the 12 months but did not reach normal values, and the median score for the physical role domain in a quality of life survey revealed a level one third that of the normal population at 12 months; both these findings suggest generalised muscle weakness. The investigators went on to postulate that the observed muscle wasting and weakness in ARDS survivors is multifactorial and may be due in part to corticosteroid-induced and critical illness-associated polyneuromyopathy. In the cohort of SARS survivors studied by Hui et al the mean 6 minute walk distance, although improved from 3 to 6 months, remained lower than the normal range across all ages at 6 months. The measured SF36 scores were also lower than normal at 6 months. The pattern of residual functional impairment in post-SARS patients at 6 months therefore appears to follow that seen in post-ARDS patients. Although SARS patients might not have suffered from critical illnessassociated polyneuromyopathy, other factors such as corticosteroid-induced myopathy, prolonged confinement and/ or immobilisation and SARS-induced myositis, as postulated by Hui et al, are likely contributors to post-SARS generalised muscle weakness. It is worthwhile emphasising that SARS is a multisystem disease involving not only the lungs but also the gastrointestinal tract, liver, blood clotting system, ${ }^{12}$ and the muscles; the pathogenesis may be by direct viral attack and/or by cytokine mediation. Muscle involvement is evidenced by myalgia reported in $50.8 \%$ of

${ }^{*} \mathrm{Ng}$ et $\mathrm{a}^{2}$ adopted a European reference range for their lung function laboratory, unlike the Singaporean reference range adopted by Hui et al' (personal communication with $\mathrm{Dr}$ Johnny Chan, Queen Elizabeth Hospital, Hong Kong, December 2004). 
the Hong Kong SARS cohort ${ }^{13}$ and by raised creatinine kinase levels found in $32.1 \%$ of the patients studied by Hui et al. ${ }^{11} \mathrm{~A}$ much rarer but more severe form of muscle involvement in SARS-rhabdomyolysis-has also been reported. ${ }^{14}$ The multifactorial generalised muscle weakness found at 6 months, extrapolated from the ARDS experience, will probably persist beyond 6 months but most patients will eventually be able to resume full time work. $^{8}$

\section{PSYCHOLOGICAL RECOVERY}

Not only should we consider the functional recovery pathway of post-SARS patients, we should also be concerned with their psychological recovery pathway. In a recent review of the long term outcomes after critical illness, Herridge described not only physical dysfunction in ARDS survivors but also psychological dysfunction following ARDS, including both neurocognitive impairment such as memory deficit and impaired attention and concentration, and psychological impairment such as depression and post-traumatic stress disorder. ${ }^{15}{ }^{16}$ Patients with SARS during the 2003 outbreak no doubt went through a highly stressful experience, including their close encounter with a then mysterious infectious disease which, in a minority of patients, could be fatal, and the physical and social isolation mandated by the authority. The psychological impact of the SARS episode may mirror that of a critical illness in many ways.

The efforts of Hui et al and Ng et al in systematically following up post-SARS patients are laudable and will serve as a nucleus for the body of knowledge on the functional and psychological recovery pathway of post-SARS patients.

Thorax 2005;60:361-362.

doi: $10.1136 /$ thx.2004.035972

Correspondence to: Dr J C K Chan, Consultant (Medical Development), Hospital Authority Head Office, 147B Argyle Street, Hong Kong; chancki@ha.org.hk

\section{REFERENCES}

1 Hui DS, Joynt GM, Wong KT, et al. Impact of severe acute respiratory syndrome (SARS) on pulmonary function, functional capacity and quality of life in a cohort of survivors. Thorax 2005;60:401-9.

$2 \mathrm{Ng} \mathrm{CK}$, Chan JWM, Kwan TL, et al. Six month radiological and physiological outcomes in severe acute respiratory syndrome (SARS) survivors. Thorax 2004;59:889-91.

3 Herridge MS, Cheung AM, Tansey CM, et al. One-year outcomes in survivors of the acute respiratory distress syndrome. N Engl J Med 2003;348:683-93.

4 Neff TA, Stocker R, Frey HR, et al. Long-term assessment of lung function in survivors of severe ARDS. Chest 2003;123:845-53.

5 Orme J Jr, Romney JS, Hopkins RO, et al. Pulmonary function and health-related quality of life in survivors of acute respiratory distress syndrome. Am J Respir Crit Care Med 2003;167:690-4.

6 Suntharalingam G, Regan K, Keogh BF, et al. Influence of direct and indirect etiology on acute outcome and 6-month functional recovery in acute respiratory distress syndrome. Crit Care Med 2001;29:562-6.

7 Rothenhausler HB, Ehrentraut S, Stoll C, et al. The relationship between cognitive performance and employment and health status in long-term survivors of the acute respiratory distress syndrome: results of an exploratory study. Gen Hosp Psychiatry 2001 ; 23:90-6.

8 Schelling G, Stoll C, Vogelmeier C, et al. Pulmonary function and health-related quality of life in a sample of long-term survivors of the acute respiratory distress syndrome. Intensive Care Med 2000;26:1304-11

9 Angus DC, Musthafa AA, Clermont G, et al. Quality-adjusted survival in the first year after the acute respiratory distress syndrome. Am J Respir Crit Care Med 2001;163:1389-94.

10 Rubenfeld GD. Is SARS just ARDS? JAMA 2003;290:397-9.

11 Lee N, Hui DS, Wu A, et al. A major outbreak of severe acute respiratory syndrome in Hong Kong. N Engl J Med 2003;348:1986-94.

12 Sung JJY, Wong RSM, Chan JCK. Clinical course of severe acute respiratory syndrome. In: Chan JCK, Wong VCW, eds. Challenges of severe acute respiratory syndrome. Oxford: Elsevier, 2005(in press).

13 Donnelly CA, Ghani AC, Leung GM, et al. Epidemiological determinants of spread of causal agent of severe acute respiratory syndrome in Hong Kong. Lancet 2003;361:1761-6

14 Wang JL, Wang JT, Yu CJ, et al. Rhabdomyolysis associated with probable SARS. Am J Med 2003;115:421-2.

15 Herridge MS. Long-term outcomes after critical illness. Curr Opin Crit Care 2002;8:331-6.

16 Gilleran SN, Trow TK. Long-term follow-up in survivors of acute respiratory distress syndrome. Clin Pulm Med 2003;10:241-2.

\section{Circulating endothelial progenitor cells in pulmonary inflammation}

\section{M Doerschuk}

\section{Do endothelial progenitor cells contribute to lung repair and, if so, how?}

U nderstanding how circulating stem cells released from the haematopoietic compartment accumulate and differentiate into the parenchymal cells of organs has become an exciting, thought provoking, and intriguing forefront of medical science. Many investigators have begun to address the ability of various populations of stem cells to aid in the repair of nearly every organ including the lungs, ${ }^{1-19}$ either through recruitment and differentiation into parenchymal cells or through facilitating proliferation and differentiation of cells already present to mediate the repair. Numerous questions remain about if and how stem cells can facilitate organ repair.

\section{ROLE OF ENDOTHELIAL PROGENITOR CELLS IN LUNG REPAIR}

The studies presented by Yamada and colleagues $^{1}$ in this issue of Thorax address an important aspect about endothelial progenitor cells (EPC) in lung repair. They show that patients with pneumonia confined to one lobe and no other illnesses have circulating EPC in their blood within the first day of illness, and that this number is decreased 8 weeks after treatment and recovery. Their data provide strong evidence that inflammation in the lungs induces release of progenitor cells from the bone marrow that are capable of differentiating toward endothelial cell phenotypes upon culturing in appropriate growth media. Most curiously, the patients who had fibrotic changes persisting at 8 weeks were the ones with low numbers of circulating EPC within the first 28 hours of pneumonia. These data raise the possibility that circulating EPC may contribute to normal lung repair.

As with all good clinical studies, these results raise more questions than they answer. Are these circulating EPC retained within the lungs and what is their fate? Do they participate in the repair of the lungs and, if so, what role do they play? Studies examining the lungs of patients with transplanted haematopoietic stem cells from a donor of a different sex than the recipient have proved to be helpful in showing that donor-derived endothelial and epithelial cells are present within the lungs, and many studies in other species have also supported this concept. ${ }^{20} 21$ In healthy lungs there may be little need for stem cells to contribute to the turnover rates 
of parenchymal cells, as this turnover is quite slow and may occur through relatively undifferentiated cells within the lung tissue. In mice the incorporation and differentiation of marrowderived stem cells into lung parenchymal cells is also, at best, very slow. However, when the lung is injured, the role of circulating stem cells probably becomes quite different. Yamada and other investigators have previously shown in mice that stem cells are required for the normal repair of lung tissue following pneumonia induced by lipopolysaccharide. ${ }^{2}$ Intra-airway lipopolysaccharide induced release of bone marrow-derived progenitor stem cells and their accumulation within the lungs, where they develop features of an endothelial cell phenotype. In elastase-induced emphysema, cells derived from the bone marrow develop characteristics of endothelial cells and contribute to repair of the alveolar capillary wall. $^{3}$ Differentiation of marrowderived stem cells toward epithelial cell phenotypes also occurs in this region. ${ }^{2-4}$ Studies focused on vascular repair in other organs suggest that stem cells have a role in the repair through inducing endogenous vascular proliferation and are not the only cells contributing to the new vasculature. Thus, there appear to be several roles for progenitor cells in repair of the lungs and other tissues.

Important questions remain unsolved regarding the environment and the mechanisms through which progenitor cells accumulate, differentiate, and participate in lung repair. The lungs are ideally positioned to trap the progenitor cells, since the bone marrow sinusoids empty into the venous circulation and the pulmonary microvasculature is the first capillary bed through which these cells must pass. The narrow diameter of the capillaries compared with the size of most leucocytes and stem cells suggests that their trafficking through the pulmonary capillary bed will require considerable time and deformation. ${ }^{22} 23$ Long transit times through the pulmonary capillaries may allow stem cells to sample the microenvironment and determine whether pneumonia or other lung injuries are present and repair is needed. Adhesion molecules may also play a role in the retention of these cells, and complex signalling mechanisms between chemokines and adhesion molecules are probably required, similar to the paradigms for retention of leucocytes in the lungs. Interactions between SDF-1 and CXCR4 as well as integrins, immunoglobulin-like adhesion molecules, and selectins may be required.

Once the cells are retained at the site of pulmonary inflammation, questions focus on how they differentiate toward parenchymal cell phenotypes. Studies by Ishizawa et $a l^{4}$ have suggested that hepatocyte growth factor plays an important role in the proliferation of endothelial cells in the lungs of mice with elastase-induced emphysema, suggesting that this growth factor is important in the process of lung repair. Other traditional and novel growth factors are also likely to contribute, and understanding the signalling pathways underlying differentiation and the features of the microenvironment that enhance differentiation and lung repair would be valuable and provide potential therapeutic options. Furthermore, if these progenitor cells are not themselves differentiating and contributing to repair, then understanding how they alter the microenvironment and regulate repair would also be critical to our understanding of lung disease.

Another interesting question raised by the work of Yamada et al is which parenchymal cells need to be replaced by circulating progenitor cells and whether replacing only the endothelial cells can enhance the repair and resolution of inflammation. One might hypothesise that differentiation of the progenitor cells towards an endothelial cell phenotype might be sufficient for providing the structure necessary for repair of the alveolar walls. The spotty and focal differentiation of progenitor cells in recovering inflammatory lesions $^{23}$ suggests that replacement of damaged endothelial cells may be sufficient to help with the repair. Other critical questions include the degree of differentiation that is necessary for functional repair; how similar must EPCs become to endogenous lung capillary endothelial cells to facilitate repair?

Finally, questions remain regarding the mechanisms through which progenitor cells are released from the bone marrow during the inflammatory process. The authors found an association between the numbers of circulating monocytes and lymphocytes and the numbers of circulating EPC, leading them to suggest that EPC are released through similar mechanisms as these leucocytes. Questions about the molecules that mediate release of EPC and whether these mechanisms are, in fact, common to other leucocytes beg further study. Our understanding of the mechanisms through which cells are released from the bone marrow is minimal, despite contributions from many outstanding scientists. How mechanisms thought to retain cells within the bone marrow work and how these mechanisms of retention are balanced by stimuli for release are unclear. While many commonalities are likely to exist, specific features that may be responsible for the release of one population over another might prove very beneficial and form the basis of a therapeutic intervention. Surprising effects of vascular endothelial growth factor 1 and of inhibitors of HMG-CoA reductase in inducing marrow release of EPCs are harbingers of observations likely to appear. ${ }^{24-26}$

\section{IMPLICATIONS FOR THE FUTURE}

The critical question put forward by this paper and others is therefore-what role do circulating EPCs and other progenitor cells play in the resolution of pneumonia and other pulmonary inflammatory events? Yamada et al ${ }^{1}$ have successfully translated their initial studies in mice to provide data showing that pneumonia increases the number of circulating EPC and tantalising observations that these EPC are important in effective lung repair. If a role for progenitor cells can be demonstrated in the repopulation of lung parenchymal cells themselves or in regulating the differentiation of other cells and the resolution of injury, then efforts to understand these mechanisms become critical. Differential release of progenitor cells from the bone marrow without enhancing the release of neutrophils and monocytes-which may act to damage the lung once host defence mechanisms have destroyed the offending organism-have an extraordinary therapeutic potential. Ways to manipulate the microenvironment to enhance lung repair, particularly in patients who are immunocompromised and have inadequate bone marrow responses, would have obvious therapeutic benefits. Determining which of the myriad of progenitor cells are critical in repairing lung injuries could lead to important approaches of cell based treatments where effective progenitor cells were infused in patients with acute and chronic inflammatory diseases, pulmonary hypertension, or other lung diseases. Modifying the function of these progenitor cells through gene therapy may expand their potential uses. Furthermore, understanding how to modify differentiation might also prove beneficial in diseases such as interstitial fibrosis where preventing abnormal fibrotic tissue and enhancing formation of gas exchanging tissue with normal compliance might benefit patients. Finally, one could even envisage growth of new normal lung tissue in patients with a number of lung diseases. All of us would no doubt welcome the day when our biggest problem in pulmonary disease is abuse of lung regeneration therapy by athletes who want to run, skate, jump, swim, 
bicycle, or ski better without limitations of gas exchange!

Thorax 2005;60:362-364.

doi: $10.1136 /$ thx.2004.037796

Correspondence to: $\operatorname{Dr} C$ M Doerschuk, Case Western Reserve University, Room RBC 787 , 11100 Euclid Avenue, Cleveland, $\mathrm{OH}$ 44106, USA; cmd22@case.edu

\section{REFERENCES}

1 Yamada M, Kubo H, Ishizawa K, et al. Increased circulating endothelial progenitor cells in patients with bacterial pneumonia: evidence that bone marrow derived cells contribute to lung repair. Thorax 2005;60:410-3.

2 Yamada M, Kubo H, Kobayashi S, et al. Bone marrow-derived progenitor cells are important for lung repair after lipopolysaccharide-induced lung injury. J Immunol 2004;172:1266-72.

3 Ishizawa K, Kubo H, Yamada M, et al. Bone marrow-derived cells contribute to lung regeneration after elastase-induced pulmonary emphysema. FEBS Lett 2004;556:249-52.

4 Ishizawa K, Kubo H, Yamada M et al. Hepatocyte growth factor induces angiogenesis in injured lungs through mobilizing endothelial progenitor cells. Biochem Biophys Res Commun 2004;324:276-80.

5 Iwami Y, Masuda H, Asahara T. Endothelial progenitor cells: past, state of the art, and future. J Cell Mol Med 2004;8:488-97.

6 Hristov M, Weber C. Endothelial progenitor cells: characterization, pathophysiology, and possible clinical relevance. J Cell Mol Med 2004;8:498-508.
7 Rumpold H, Wolf D, Koeck R, et al. Endothelia progenitor cells: a source for therapeutic vasculogenesis? J Cell Mol Med 2004;8:509-18.

8 Summer R, Kotton DN, Sun X, et al. Origin and phenotype of lung side population cells. Am J Physiol Lung Cell Mol Physiol 2004;287:L477-83.

9 Kotton DN, Summer R, Fine A. Lung stem cells: new paradigms. Exp Hematol 2004;43:340-3.

10 Fine A. Marrow cells as progenitors of lung tissue. Blood Cells Mol Dis 2004;32:95-6.

11 Neuringer IP, Randell SH. Stem cells and repair of lung injuries. Respir Res 2004;5:6.

12 Herzog EL, Chai L, Krause DS. Plasticity of marrow-derived stem cells. Blood 2003;102:3483-93.

13 Wang G, Bunnell BA, Painter RG, et al. Adult stem cells from bone marrow stroma differentiate into airway epithelial cells: potential therapy for cystic fibrosis. PNAS 2005; 102:186-91.

14 Reynolds SD, Giangreco A, Hong KU, et al. Airway injury in lung disease pathophysiology: selective depletion of airway stem and progenitor cell pools potentiates lung inflammation and alveolar dysfunction. Am J Physiol Lung Cell Mol Physiol 2004;287:L1256-65.

15 Kotton DN, Fine A. Derivation of lung epithelium from bone marrow cells. Cytotherapy 2003;5:169-73

16 Abe S, Lauby G, Boyer C, et al. Transplanted BM and $B M$ side population cells contribute progeny to the lung and liver in irradiated mice. Cytotherapy 2003:5:523-33.

17 Anjos-Afonso F, Siapati EK, Bonnet D. In vivo contribution of murine mesenchymal stem cells into multiple cell-types under minimal damage conditions. I Cell Sci 2004; 117:5655-64.

\section{BOHRF guidelines for occupational asthma}

\section{A J Newman Taylor, P Cullinan, P S Burge, P Nicholson, C Boyle}

\section{Publication of the first evidence based guidelines for occupational asthma}

$\mathrm{N}$ ew guidelines for the identification, management, and prevention of occupational asthma are published this month in Occupational and Environmental Medicine. ${ }^{1}$ The first evidence based guidelines for occupational asthma, they were prepared by a working group that included clinicians, patients, occupational hygienists, and representatives of the Health and Safety Executive. The work was supported by a grant from the British Occupational Health Research Foundation (BOHRF). The guidelines will be supplemented by an abbreviated version for primary care practitioners, occupational health practitioners, employers, employees, and workplace safety representatives.
These guidelines are intended to increase awareness and improve the management of occupational asthma by all practitioners who encounter such patients, and to stimulate the means to reduce its incidence by those able to effect this.

The important issues in occupational asthma concern its aetiology, diagnosis, outcome and prevention. Questions about these are not readily answered by randomised controlled trials (RCTs) and, arguably, conventional hierarchies with the RCT at the apex are not appropriate for assessing the strength of evidence used in the generation of guidelines. $^{2}$ Although not having the high internal validity of the RCT, strong
18 Takahashi $M$, Nakamura $T$, Toba $T$, et al Transplantation of endothelial progenitor cells into the lung to alleviate pulmonary hypertension in dogs. Tissue Eng 2004;10:771-9.

19 Mattsson J, Jansson M, Wernerson A, et al. Lung epithelial cells and type II pneumocytes of donor origin after allogeneic hematopoietic stem cell transplantation. Transplantation 2004;78:154-7

20 Suratt BT, Cool CD, Seris AE, et al. Human pulmonary chimerism after hematopoietic stem cell transplantation. Am J Respir Crit Care Med 2003;168:318-55.

21 Kotton DS, Ma BY, Cardoso WV, et al. Bone marrow-derived cells as progenitors of lung alveolar epithelium. Development 2001; 128:5181-8.

22 Doerschuk CM, Beyers N, Coxson HO, et al Comparison of neutrophil and capillary diameters and their relation to neutrophil sequestration in the lung. J Appl Physiol 1993;74:3040-5.

23 Wiggs BR, English D, Quinlan WM, et al. The contributions of capillary pathway size and neutrophil deformability to neutrophil transi through rabbit lungs. J Appl Physiol 1994;77:463-70.

24 Kalka C Masuda H, Takahashi T, et al. Vascular endothelial growth factor 165 gene transfer augments circulating endothelial progenitor cells in human subjects. Circ Res 2000;86:1 198-202.

25 Dimmeler S, Aicher A, Vasa M, et al. HMG-CoAreductase inhibitors (statins) increase endothelial progenitor cells via the PI3 kinase/Akt pathway. $J$ Clin Invest 2001;108:391-7.

26 Llevadot J, Murasawa S, Kureishi Y, et al. HMG CoA reductase inhibitor mobilizes bone-marrow derived endothelial progenitor cells. J Clin Invest 2001; 108:399-405. inferences can be drawn from observational studies (whose external validity can be greater than that of an RCT) when these are well designed and their findings consistent and plausible.

The guidelines address several questions that are of key importance to respiratory physicians:

- What proportion of asthma in adult life is attributable to occupation?

- What are the most frequent causes of occupational asthma and in which occupations are they encountered?

- What methods are most useful in the diagnosis of occupational asthma?

- How is a case of occupational asthma best managed?

- What is the prognosis of occupational asthma and what factors influence this?

\section{WHAT IS OCCUPATIONAL ASTHMA?}

Asthma can be aggravated or induced by an agent inhaled at work. "Occupational asthma" is generally limited to asthma induced by a workplace agent, either following the inhalation of an irritant chemical in toxic concentration (irritant induced occupational asthma) or, more commonly, as the outcome of a hypersensitivity reaction to an inhaled protein 
Table 1 Agents responsible for more than 100 cases of occupational asthma (1992-7) and the occupations in which they were encountered $^{3}$

\begin{tabular}{ll}
\hline Occupation & Agent \\
\hline Spray painter & Isocyanates \\
Baker & Flour \\
Wood worker & Wood \\
$\begin{array}{l}\text { Nurse } \\
\text { Laboratory technician, } \\
\text { scientist and assistant } \\
\begin{array}{l}\text { Welder, solderer or } \\
\text { electrical assembly }\end{array}\end{array}$ & Laboratory animals \\
\hline
\end{tabular}

allergen or chemical (hypersensitivity induced occupational asthma). Some $90 \%$ of cases of occupational asthma are hypersensitivity induced and the guidelines primarily address this group.

Irritant induced asthma typically develops within hours of an acute inhalation incident; hypersensitivity induced asthma only develops after a latent interval usually of several months-but occasionally weeks or years - of asymptomatic exposure. In some cases, usually when caused by inhaled proteins but also by some low molecular weight chemicals, hypersensitivity induced occupational asthma is accompanied by the development of specific IgE antibody which can be identified in the blood or by a skin prick test.

\section{HOW FREQUENT IS OCCUPATIONAL ASTHMA AND IN WHOM DOES IT OCCUR?}

Estimates of the incidence of occupational asthma by occupation and the agents identified as causative in the UK during the past 15 years have come from the Surveillance of Work and Occupational Respiratory Disease (SWORD) scheme to which chest physicians and occupational physicians have regularly reported new cases of occupational lung disease. The results of SWORD have been surprisingly consistent, with occupational asthma the single most frequently reported condition-accounting for some $25 \%$ of reported cases with an estimated average of 2000 new cases each year. These cases represent only those seen by chest and occupational physicians in whom the attribution of asthma to work is made and reported. Interestingly, with the exception of the rise and fall of asthma attributed to latex allergy during the 1990s, the other causes of occupational asthma and the occupations in which the cases were employed have remained surprisingly stable during this period. The agents identified as responsible for more than 100 cases between
1992 and $1997^{3}$ and the occupations in which they were encountered are shown in table 1 .

Several recent studies, many using data from the European Community Respiratory Health Survey, have estimated the proportion of cases of asthma in those of working age to which occupational factors have contributed. In essence, such studies estimate the relative frequency of different occupations in (usually) prevalent cases of asthma, comparing these with an occupational grouping considered not to have an increased risk from occupational cause. Two meta-analyses of these studies have recently been reported. The first suggested a median population attributable risk (PAR) of $9 \%$ for all studies and 15\% for the highest quality studies; the second a PAR of $15 \%$. The implication of these observations is that between 1 in 10 and 1 in 7 cases of asthma in adult life are, at least in part, attributable to occupation.

\section{WHY MAKE THE DIAGNOSIS OF OCCUPATIONAL ASTHMA?}

Many studies reported in the past 25 years have found that symptoms of asthma and airway hyperresponsiveness persist in a high proportion of cases (between a third and a half) followed up for several years after avoidance of exposure to the cause, which includes isocyanates, wood dusts, acid anhydrides, colophony, and snow crab protein. Although the majority of these studies followed up patients seen in hospital and are therefore likely to reflect more severe disease and those with continuing symptoms, they are consistent in indicating continuing disease in a significant proportion of cases. In addition, studies of social and financial consequences indicate that about one third of cases remain unemployed up to 5 years from diagnosis and suffer consequent financial disadvantage.

Several studies have identified common factors which increase the probability of a favourable prognosis. These include avoidance of further exposure to the causal agent; relatively normal lung function at the time of diagnosis; shorter duration of symptoms before diagnosis; and shorter duration of symptoms before avoidance of exposure. By implication, as emphasised in the guidelines, the resolution of symptoms and the restoration of normal lung function are most likely in patients whose occupational asthma is identified early in the course of the disease and who avoid exposure within a short interval from the onset of symptoms, ideally within a few months.

The high proportion of cases remaining unemployed after several years suggests that the value of a compensation scheme for occupational asthma could lie in supporting retraining, enabling return to the labour market. There is limited evidence from France and Canada to support this.

\section{HOW BEST TO MAKE THE DIAGNOSIS OF OCCUPATIONAL ASTHMA?}

The diagnosis of occupational asthma is an iterative process. The components that show the greatest diagnostic utility are: a history of work related symptoms; serial peak flow measurements; and, where applicable and available, identification of specific IgE antibody in the serum or by skin prick test. The gold standard for the diagnosis of occupational asthma is bronchial provocation testing which is indicated in a minority of cases and is of limited availability in the UK.

Sensitisation and the symptoms of occupational asthma due to the majority of causes of occupational asthma are most likely to develop in the first few years of exposure. Rhinitis accompanies IgE associated occupational asthma in a high proportion of cases and generally develops about a year before the onset of symptoms.

Because of the potential implications of occupational asthma for future employment, the guidelines recommend that the diagnosis should be based on objective criteria (functional, immunological, or both) and that suspected cases should be referred to a physician with expertise in the disease.

A history of symptoms of asthma which improve on days away from work, both by questionnaire and taken by experts, has a high sensitivity (few false negatives) but relatively low specificity (a high proportion of false positives). Objective tests are needed to increase the specificity of the diagnosis. Studies of serial peak flow measurements undertaken in a clinical setting have shown high sensitivity and specificity provided a minimum of four readings per day are made. Both visual analysis by experts and computed analysis show good diagnostic performance.

Pre and post shift measurements of lung function are of limited value and cannot be recommended in the diagnosis of occupational asthma. Increased airway responsiveness (using different methods) has been found in many cases of occupational asthma, but there are also many reports of normal airway responsiveness to inhaled histamine or methacholine within 24 hours of exposure in workers with confirmed occupational asthma. Airway responsiveness even within 24 hours of exposure is 
not a sufficiently sensitive test and a negative result does not exclude a diagnosis of occupational asthma.

Specific IgE antibody is associated particularly with cases of occupational asthma caused by high molecular weight allergens such as enzymes, flour, latex, and laboratory animal urine proteins, but also with asthma caused by some low molecular weight chemical sensitisers including complex platinum salts, acid anhydrides, and some reactive dyes. Specific IgE confirms sensitisation to an agent encountered at work but, alone, does not confirm the diagnosis of occupational asthma. In general, specific IgE is very sensitive (few false negatives) but non-specific (high false positive rate). It therefore has more power in excluding an agent as a cause of occupational asthma than in identifying it as the cause.

\section{HOW IS OCCUPATIONAL ASTHMA BEST MANAGED?}

Because the risk of sensitisation and associated occupational asthma cannot be eliminated in those exposed to its causes, and the prognosis is best when the disease is identified and exposure avoided early, regular health surveillance is recommended for all workers where a residual risk of occupational asthma is identified.

Health surveillance should probably be undertaken at least annually and, because the risk of sensitisation and associated asthma is highest during the early years of exposure, more frequently in the first 2 years of exposure. Evidence for the effectiveness of health surveillance for occupational asthma is, however, limited to one study of isocyanate workers in Ontario, Canada where regular surveillance was combined with improved control of isocyanate exposure, making it difficult to dissociate their separate effects. Nonetheless, as would be anticipated, more cases of isocyanate asthma were identified initially; subsequently, in association with a reduced incidence, cases were identified sooner after the onset of symptoms with better lung function and a better outcome.

The implications for respiratory physicians are that they should endeavour to see and diagnose cases of occupational asthma within as short a time as practicable from the onset of symptoms and consider the potential for future employment with the patients' employers and their occupational health advisers.

\section{HOW CAN OCCUPATIONAL ASTHMA BEST BE PREVENTED?}

Although there is evidence for individual susceptibility-both genetic (atopy, HLA phenotype) and behavioural (smoking) - to several of the causes of occupational asthma, none is sufficiently discriminating to be appropriate as a basis for pre-employment screening. Studies of laboratory animal workers, bakers, enzyme detergent, platinum refinery and acid anhydride workers have documented gradients of increasing risk of sensitisation and asthma with increasing intensity of exposure, implying that the incidence of the disease would be reduced by a reduction in the level of exposure. The results of intervention studies have been reported: in a limited number of circumstances a reduction in the airborne concentrations of enzymes in the detergent industry, of latex in hospitals, and isocyanates in various workplaces have been followed by reduced incidence of disease.

\section{CONCLUSION}

It is hoped these guidelines will draw the attention of respiratory physicians and other health professionals to the importance of occupation as a cause of asthma in adult life, to the importance of accurate diagnosis at an early and potentially remediable stage of the disease, and to the need for communication between respiratory, primary care and occupational health practitioners and their patients' employers for optimal management of patients with occupational asthma.

Thorax 2005;60:364-366.

doi: $10.1136 /$ thx.2004.032367

\section{Authors' affiliations}

A Newman Taylor, P Cullinan, Department of Occupational \& Environmental Medicine, $\mathrm{NHLl}$ at Imperial College Faculty of Medicine, London SW3 6LR, UK

P S Burge, Birmingham Heartlands Hospital, Bordesley Green East, Birmingham B9 5SS, UK P Nicholson, P\&G, Whitehall Lane, Egham, Surrey TW20 9NW, UK

C Boyle, Industrial Chemicals Unit, Health and Safety Executive, Magdalen House, Stanley Precinct, Bootle, Merseyside L20 3QZ, UK

Correspondence to: Professor A J Newman Taylor, Department of Occupational \& Environmental Medicine, NHLI at Imperial College Faculty of Medicine, Emmanuel Kaye Building, Ib Manresa Road, London SW3 6LR, UK; e.haining@rbh.nthames.nhs.uk

\section{REFERENCES}

1 Nicholson PJ, Cullinan P, Newman Taylor AJ, et al. Evidence based guidelines for the prevention, identification and management of occupational asthma. Occup Environ Med 2005;62:290-9.

2 Glasziou P, Vandenbroucke J, Chalmers. Assessing the quality of research. BMJ 2004;328:39-41.

3 McDonald JC, Keynes HL, Meredith SK. Reported incidence of occupational asthma in the United Kingdom, 1989-1997. Occup Environ Med 2000;57:823-9. 